\title{
A narrative review of research advances in mesenchymal stem cell therapy for asthma
}

\author{
Xiu Yu, Li Yu, Bingxin Guo, Rongchang Chen, Chen Qiu \\ Department of Respiratory and Critical Care Medicine, Shenzhen People's Hospital (Second Clinical Medical College of Jinan University \& First \\ Affiliated Hospital of Southern University of Science and Technology), Shenzhen Institute of Respiratory Diseases, Shenzhen, China \\ Contributions: (I) Conception and design: C Qiu, R Chen; (II) Administrative support: C Qiu, R Chen; (III) Provision of study materials or patients: X \\ Yu, L Yu, B Guo; (IV) Collection and assembly of data: X Yu, L Yu, B Guo; (V) Data analysis and interpretation: X Yu, L Yu, B Guo; (VI) Manuscript \\ writing: All authors; (VII) Final approval of manuscript: All authors. \\ Correspondence to: Rongchang Chen; Chen Qiu. Department of Respiratory and Critical Care Medicine, Shenzhen People's Hospital (Second \\ Clinical Medical College of Jinan University \& First Affiliated Hospital of Southern University of Science and Technology), Shenzhen Institute of \\ Respiratory Diseases, Shenzhen 518020, China. Email: chenrc@vip.163.com; qiuchen@email.jnu.edu.cn.
}

\begin{abstract}
Asthma is a chronic inflammatory disease of the airways that involves multiple cells, including inflammatory cells, structural cells, and cellular components. Glucocorticoids and beta-receptor agonists are still the first choices for asthma treatment. However, the asthma symptoms may still be poorly controlled in some patients after an optimal treatment. Mesenchymal stem cells (MSCs) are characterized by the potential for multi-directional differentiation and can exert immunomodulatory and anti-inflammatory effects. Its role in treating asthma has increasingly been recognized in recent years. In this review article, we sought to summarize the recent advances in the therapeutic effects of MSCs on several types of asthma and explain the relevant mechanisms. Articles on asthma treatment with MSCs as of January 2020 were searched in PubMed, Google Scholar, and Web of Science databases. It was found that MSCs have therapeutic effects on allergic asthma, non-allergic asthma and occupational asthma; gene-modified or pretreated MSCs improves the therapeutic effects of MSCs in asthma; MSC-derived conditioned medium or extracellular vesicles possess the considerable curative effect as MSC on asthma; and MSCs exert their therapeutic effects on asthma by restoring Th1/Th2 balance, reversing Th17/Tregs imbalance, inhibiting DC maturation, and promoting the switch of M1 to M2 and repairing epithelial injury. Thus, MSCs may be a promising treatment for asthma.
\end{abstract}

Keywords: Mesenchymal stem cells (MSCs); asthma; treatment; mechanism

Submitted Jun 30, 2020. Accepted for publication Oct 24, 2020.

doi: 10.21037/atm-20-6389

View this article at: http://dx.doi.org/10.21037/atm-20-6389

\section{Introduction}

Asthma (or bronchial asthma) is a clinically common and heterogeneous disease, with airway inflammation, hyperresponsiveness, and remodeling as its main pathological features. Its typical clinical manifestations include wheezing, shortness of breath, chest tightness, and cough, which can be accompanied by expiratory airflow limitation $(1,2)$. There are 300 million people affected by asthma worldwide. The prevalence rate of asthma ranges from $1-18 \%$, and the mortality is rising annually (3). With the accelerated global industrialization and environmental pollution, over 400 million people may live with asthma by 2025 , posing a severe threat to public health (4). At present, anti-inflammatory drugs (e.g., glucocorticoids) and bronchodilators (e.g., $\beta$-adrenoreceptor agonists) remain the first choice to treat asthma $(5,6)$. However, the asthma symptoms are still poorly controlled after an optimal treatment in $5 \%$ to $10 \%$ of asthma patients. The cost of refractory asthma therapy contributes to approximately $50 \%$ of all of asthma, placing a significant financial burden on individuals, families, and society (7). Therefore, new 
treatment strategies and directions are essential for the treatment and management of asthma patients.

Mesenchymal stem cells (MSCs) are adult stem cells that express stem cell markers, including CD105, CD73, CD90, CD44, and CD29, and are negative for hematopoietic antigens including CD45, CD34, CD14, and CD11b. They can differentiate into osteocytes, chondrocytes, and adipocytes $(8,9)$. Many studies have shown that MSCs are endowed with remarkable antiinflammatory and immunoregulatory properties and have the potential to repair the damaged tissues; thus, they can treat numerous diseases such as hematological disease, organ transplantation, diabetes, graft-versus-host disease, inflammatory diseases, as well as neurological, bone and cartilage, cardiovascular, and autoimmune diseases and so on (10). During recent years, there have been many reports regarding MSC therapies for asthma in the laboratory. But until now there is not clinical study of MSC-based therapies for asthma.

This article aims to review the roles and mechanisms of MSCs in the treatment of asthma, including the therapeutic effect of MSC from different sources on asthma; the therapeutic effect of MSC on different asthma; the therapeutic effect of modified MSCs on asthma; the underlying mechanisms MSC exerting the therapeutic effect of asthma. We focused on the therapeutic effects of MSC on asthma and the difficulties of applying MSC in the clinical treatment of asthma. We present the following article in accordance with the Narrative Review reporting checklist (available at http:// dx.doi.org/10.21037/atm-20-6389).

\section{Methods}

Articles on asthma treatment with MSCs as of January 2020 were searched in PubMed, Google Scholar, and Web of Science databases. The subject terms used included "Mesenchymal stem cells and asthma," "Mesenchymal stem cells and airway inflammation," and "Mesenchymal stem cells and airway remodeling," as the retrieved articles were further analyzed.

\section{Discussion}

\section{Therapeutic effects of MSCs on asthma}

Asthma is a heterogeneous disease (1). Its specific types include allergic and non-allergic asthma, occupational asthma, exercise-induced asthma, and cough-variant asthma (11). It can also be divided into eosinophil or neutrophil types according to inflammatory cell types by immunopathogenesis (12). Among them, allergic asthma is mostly manifested as eosinophil infiltration, while neutrophil infiltration often features non-allergic asthma and can quickly progress into severe refractory asthma. In animal models of asthma, ovalbumin (OVA), dust mites, or cockroach extracts (CREs) typically is used to induce allergic asthma. Meanwhile, non-allergic asthma is induced by coadministration of OVA with lipopolysaccharide (LPS) or polyinosinic:polycytidylic acid (poly I:C) (13). Table 1 summarizes the therapeutic effects of MSCs from various sources on several types of asthma.

\section{Therapeutic effects of MSCs on allergic asthma}

Allergic asthma includes acute or chronic asthma. Acute asthma is mainly manifested as airway hyperresponsiveness (AHR) and airway inflammation, whereas airway remodeling often accompanies chronic asthma (4). Acute asthmatic mouse induced by OVA or dust mites are characterized with AHR, increase of white blood cell (WBC; especially eosinophils and lymphocytes) infiltration, enhancement of Th2 cytokines levels [including interleukin 4 (IL-4), IL-5, and IL-13] in bronchial alveolar lavage fluid (BALF) and elevation of immunoglobulin G1 (IgG1) and $\mathrm{IgE}$ in serum (32) Bone marrow-derived MSCs (BMSCs), adipose-derived MSCs (ADMSCs), human umbilical cord blood-derived MSCs (hUMSCs), and human placentaderived MSCs (hPMSCs) can alleviate AHR and inhibit increase of immune cells numbers and Th2 cytokines levels in mouse models of acute asthma to varying degrees (16-18,24,26,27,29). Besides AHR and airway inflammation, airway remodeling including thickening of the subepithelial basement membrane and airway smooth muscles layer, hyperplasia of goblet cells, subepithelial fibrosis, and collagen deposition can also be found in OVAor dust mite-induced chronic asthma models. BMSCs, compact bone-derived MSCs (CBMSCs), ADMSCs, and mesenchymoangioblast-derived MSCs (MCA-MSCs) can suppress AHR, airway inflammation, and airway remodeling to varying degrees $(14,15,17,22,23,30)$. Abreu et al. compared the therapeutic effects of MSCs from various sources and found BMSCs, ADMSCs, and lungderived MSCs (LMSCs) were differentially effective at reducing airway inflammation and remodeling and improving lung function. BMSCs remarkably produced 
Table 1 Therapeutic effects of MSCs on asthma in murine models

\begin{tabular}{|c|c|c|c|}
\hline Type of MSCs & Asthma model & Therapeutic effects & References \\
\hline BMSCs & $\begin{array}{l}\text { A mouse model of dust mite-induced } \\
\text { acute asthma }\end{array}$ & Inhibited methacholine-induced AHR and airway inflammation & $(18)$ \\
\hline BMSCs & $\begin{array}{l}\text { A rate model of TDI-induced severe } \\
\text { asthma }\end{array}$ & $\begin{array}{l}\text { Reduce the number of eosinophils, neutrophils, and goblet cells in } \\
\text { BALF and inhibited AHR and collagen deposition }\end{array}$ & (19) \\
\hline BMSCs & $\begin{array}{l}\text { A mouse model of AHE-induced } \\
\text { neutrophilic asthma }\end{array}$ & $\begin{array}{l}\text { Alleviated AHR, suppressed the increase of neutrophils in BALF, } \\
\text { and inhibited the release of Th17 cytokine }\end{array}$ & $(21)$ \\
\hline CBMSCs & $\begin{array}{l}\text { A mouse model of OVA-induced } \\
\text { chronic asthma }\end{array}$ & $\begin{array}{l}\text { Reduced the thickness of the epithelium, the smooth muscle layer, } \\
\text { and basement membrane and inhibited the hyperplasia of goblet } \\
\text { cells }\end{array}$ & $(22)$ \\
\hline mADMSCs & $\begin{array}{l}\text { A mouse model of dust mite-induced } \\
\text { chronic asthma }\end{array}$ & $\begin{array}{l}\text { Reduced the number of total white blood cells and eosinophils and } \\
\text { the serum IgE level and inhibited AHR and mucus secretion }\end{array}$ & $(23)$ \\
\hline mADMSCs & $\begin{array}{l}\text { A mouse model of OVA-induced } \\
\text { acute asthma }\end{array}$ & Inhibited AHR and airway inflammation & $(24)$ \\
\hline hPMSCs & $\begin{array}{l}\text { A mouse model of OVA- and Poly I:C- } \\
\text { induced neutrophilic asthma }\end{array}$ & $\begin{array}{l}\text { Reduced the number of neutrophils and macrophages and the } \\
\text { content of IL-5 and CXCL15 in BALF }\end{array}$ & $(28)$ \\
\hline hPMSCs & $\begin{array}{l}\text { A rat model of OVA-induced acute } \\
\text { asthma }\end{array}$ & $\begin{array}{l}\text { Improve AHR and inflammation by regulating the Th17/Treg balance } \\
\text { in rats with asthma }\end{array}$ & $(29)$ \\
\hline MCA-MSCs & $\begin{array}{l}\text { A mouse model of OVA-induced } \\
\text { chronic asthma }\end{array}$ & $\begin{array}{l}\text { Inhibited goblet cell metaplasia and AHR, reduced the number } \\
\text { of subepithelial myofibroblasts and collagen deposition and } \\
\text { decreased TGF- } \beta \text { expression in airway }\end{array}$ & $(30)$ \\
\hline iPSC-MSCs & $\begin{array}{l}\text { A mouse model of OVA- and LPS- } \\
\text { induced neutrophilic asthma }\end{array}$ & $\begin{array}{l}\text { Decreased number of neutrophils in BALF and the number of Th17 } \\
\text { and Th2 cells in lungs, lowered the levels of sIL-17A and p-STAT3 } \\
\text { and increased the number of Th1 cells in lungs }\end{array}$ & $(31)$ \\
\hline
\end{tabular}

AHE, Aspergillus fumigatus hyphal extract; AHR, airway hyper-responsiveness; BALF, bronchoalveolar lavage fluid; BMSCs, bone marrowderived MSCs; CBMSCs, compact bone-derived MSCs; hADMSCs, human adipose tissue-derived MSCs; hPMSCs, human placentaderived MSCs; hUMSCs, human umbilical cord blood-derived MSCs; iPSC-MSCs, human induced pluripotent stem cell-derived MSCs; LPS, lipopolysaccharide; mADMSCs, mouse adipose tissue-derived MSCs; OVA, ovalbumin; Poly I:C, polyinosinic-polycytidylic acid; TDI, toluene diisocyanate. 
more significant reductions in collagen fiber content in the lung parenchyma, eosinophil infiltration, and levels of IL-4, IL-13, transforming growth factor (TGF)- $\beta$, and vascular endothelial growth factor (VEGF) in lung homogenates and increased IL-10 and interferon (IFN)- $\gamma$ in lung tissue compared to AD-MSCs and LMSCs (33).

\section{Therapeutic effects of MSCs on non-allergic asthma}

Non-allergic asthma is mostly manifested as neutrophils infiltration in lung, increased Th17 cytokines, airway epithelial injury, airway remodeling, and glucocorticoid resistance. In a mouse model of asthma induced by Aspergillus fumigatus hyphal extract (AHE), Lathrop et al. found increased AHR and neutrophil infiltration, along with significantly increased IL-4, IL-17a, IL-12 (p40), keratinocytes (KC), and CC chemokine ligand 5 (CCL5) and IL-3 in BALF. Tail-vein injection of BMSCs could significantly inhibit AHE-induced AHR and neutrophil infiltration and reduce Th17 cytokines (21). In another study, hUMSCs significantly suppressed the OVA- and polyinosinic:polycytidylic acid (Poly I:C)-induced increase in numbers of neutrophils and macrophages in BALF and the production of IL-5 and CXCL15 (28). Fang et al. showed human-induced pluripotent stem cell (iPSC)derived MSCs (iPSC-MSCs) significantly reduced the OVA- and LPS-induced increase in counts of neutrophils, Th17, and Th2 cells in the lung tissues and the increase of sIL-17A and p-STAT3 levels and increased numbers of Th1 cells in the lungs (31).

\section{Therapeutic effects of MSCs on other asthma types}

Occupational asthma (OA) is characterized by airway inflammation and AHR, which leads to progressive airway remodeling and a decline in lung function. hADMSCs could significantly suppress the infiltration of neutrophils and lymphoplasmocytes, the increase of AHR, and the increase of $\operatorname{IgE}$ content in the lungs of mice with occupational asthma induced by ammonium persulfate; also, it could reduce the proliferation and hypertrophy of smooth muscle cells around the bronchioles (25). Also, in toluene diisocyanate (TDI) or ragweed-induced severe asthma models, BMSCs significantly lowered numbers of eosinophils and neutrophils and the levels of IL-4, IL-5, and IL-13 in BALF; also, it reduced the serum IgG1 and IgE levels and numbers of goblet cells and suppressed AHR and collagen deposition $(19,20)$.

\section{Therapeutic effects of gene-modified or -pretreated MSCs on asthma}

The roles of genetic modification of MSCs therapies for asthma have increasingly been recognized in recent years. Miro1, a mitochondrial Rho-GTPase, regulates intercellular mitochondrial movement. Compared with unmodified BMSCs, overexpression of Miro1 in MSC leads to enhanced mitochondrial transfer and rescue of epithelial injury and further inhibits AHR and airway remodeling in mouse models of acute asthma induced by CRE, dust mite extract, or OVA (34). Erythropoietin (EPO) has antioxidant, antiapoptotic, and anti-inflammatory effects and can inhibit the synthesis of IL-4 and IL-5. In the OVA-induced chronic asthma mouse models, MSCs over-expressing EPO (EPOMSCs) showed a more potently suppressive effect on local asthmatic airway inflammation and the level of IL-4, IL-5, and IL-13 in lung tissue than MSCs. The numbers of goblet cells, the thicknesses of the smooth muscle layer, collagen density, counts of proliferating cell nuclear antigen-positive (PCNA+) MSC, and von Willebrand factor positive (vWF+) vessels were also significantly inhibited by EPO-MSCs (35).

More evidence has shown that exposure of MSCs to a specific inflammatory stimulus or pretreatment can enhance their immunomodulatory effects. Eicosapentaenoic acid (EPA) is an omega-3 fatty acid with immunomodulatory properties and can inhibit the inflammatory response of human asthmatic alveolar macrophages in vitro (36). Compared with untreated BMSCs, BMSCs pretreated with EPA better-inhibited bronchoconstriction, airway remodeling, airway inflammation, excessive mucus secretion, hyperplasia of the thymus, lymph nodes, and bone marrow, increased the IL-10 level in BALF and numbers of M2 macrophages in the lungs. These differences were observed in a mouse model of allergic asthma induced by house dust mite (HDM) extract, showing a better therapeutic effect. Cobalt chloride $\left(\mathrm{CoCl}_{2}\right)$ is a hypoxia-mimetic compound that induces biochemical and molecular responses like those observed under hypoxic conditions. Compared with untreated hUMSCs, $\mathrm{CoCl}_{2}$-treated hUMSCs could further suppress the airway inflammation in OVA-induced acute asthma mouse models (37). In another study, compared with unstimulated BMSCs, BMSCs stimulated by asthma serum could further lower the levels of IL-4, IL-13, and eosinophil chemokines in BALF, decrease the WBC counts in BALF, 
bone marrow, and lymph nodes, increase IL-10 level in BALF and improve lung function (38).

\section{Therapeutic effects of MSC-derived conditioned medium (MSC-CM) and extracellular vesicles (MSC-EV) on astbma}

Both MSC-CM and MSC-EV have immunomodulatory activities like MSCs and may be useful for treating asthma. Cruz et al. showed that either MSC-CM or MSC-EV was equally potent as MSCs in mitigating AHR and Th2/Th17mediated allergic lung inflammation in mouse models of AHE-induced severe refractory asthma (39). Keyhanmanesh et al. also found that repeated administration of MSC-CM reduced lung pathological injuries in OVA-induced acute asthmatic rat models (40).

\section{Therapeutic effects of the combinations of MSCs with other drugs on asthma}

The combined use of MSCs and other drugs can improve the pathological characteristics of asthma to a greater extent. Compared with MSCs alone, the combined administration of MSCs with the anti-fibrotic drug, serelaxin (RLN), can further reverse the peribronchial inflammation, collagen deposition, subepithelial fibrosis, and AHR in the OVAinduced chronic asthma mouse models (41).

\section{The molecular mechanisms of MSCs in treating asthma}

\section{Mechanisms of MSC migration}

Lung lesions can trigger the release of various chemokines, which can induce the migration and colonization of vascular MSCs towards the damaged sites and then exert an immunomodulatory effect and improve asthma symptoms (42). Transforming growth factor-beta 1 (TGF- $\beta 1$ ) is a multifunctional cytokine that plays critical roles in cell growth/differentiation and immune regulation. It can recruit MSCs to participate in tissue repair and remodeling at injured sites (43). In vitro experiments have shown that CRE-stimulated human epithelial cellconditioned medium can induce the migration of BMSCs. TGF- $\beta 1$ neutralizing antibody significantly inhibited the migration of BMSCs. In the CRE-induced chronic asthma mouse models, the migration of green fluorescent protein (GFP)-labeled BMSCs from the blood and bone marrow towards the airway was increased, and TGF- $\beta 1$ neutralizing antibody could inhibit the CRE-induced recruitment of MSCs (43). Also, either using BMSCs isolated from TGF- $\beta 1$ knockout mice or the injection of the TGF- $\beta 1$ antibody followed by BMSCs administration could not inhibit the inflammatory response in a ragweed-induced mouse asthma model (20).

Aryl hydrocarbon receptor (AhR), a receptor for many common environmental pollutants, can sense environmental and microbial pollution. In a CRE-induced acute asthma mouse model, compared with wild-type (WT) mice, AhR-deficient (AhR-/-) mice showed enhanced lung inflammation. After the MSCs labeled with GFP were intravenously injected, it was found the cockroach allergenchallenged AhR (-/-) mice displayed less migration of MSCs to the lungs compared with WT mice. Therefore, AhR affects lung inflammation in asthmatic mice by mediating the migration of BMSCs (44).

CXC motif chemokine receptor type 4 (CXCR4) can recruit MSCs towards the liver and pancreatic islets. In the OVA-induced acute allergic asthma models, the expression of CXCR4 on the surface of BMSCs was increased in the asthma group, and the expression of CXCR4 ligand SDF1 was increased in the asthmatic lung tissue, compared with the control group. The knockdown of CXCR4 in BMSCs blocked the migration of BMSCs to the lungs of asthmatic mice (16).

\section{Mechanisms of MSCs in the treatment of airway inflammation and airway remodeling in asthma models}

The excessive production of helper T lymphocyte type 2 (Th2) and Th17 cytokines promotes the eosinophil- and neutrophil-mediated airway inflammation, respectively (45). Excessive maturation of dendritic cells (DC) can further activate Th2 cells (46). The switch of macrophages into the M1 type can also aggravate the inflammatory response (47). Apoptosis and injury of epithelial cells is a major contributor to airway remodeling (48).

\section{Regulatory effect of MSCs on T cells}

Th2 dominance caused by Th1/Th2 imbalance is the primary immunological mechanism in the pathogenesis of eosinophilic asthma (26). Th1, differentiated from Th0 cells, mainly secretes IFN- $\gamma$, IL-12 and other cytokines that regulate normal immune function (17); however, the cytokines (e.g., IL-4, IL-5, and IL-13) secreted by Th2 can cause the increase of inflammatory cells and AHR and are 
involved in the pathogenesis of asthma $(16,23,26,43)$. Many studies have proved MSCs exert their therapeutic effect on asthma mainly by restoring Th1/Th2 balance, although the specific mechanism remains unclear. Goodwin found BSMCs could reduce Th2 cytokines and increase Th1 cytokines in BALF of asthmatic mice but did not inhibit the elevated Th2 cytokines in asthmatic mice lacking the IFN- $\gamma$ receptor. BMSCs rely on the IFN- $\gamma$ pathway to promote the conversion of antigen-specific CD4 $\mathrm{T}$ lymphocytes towards Th1 and thus inhibit Th2-mediated allergic airway inflammation (17).

The imbalance between Th17 and regulatory $\mathrm{T}$ cells (Tregs) is an important mechanism that mediates the recruitment of neutrophils to the lungs and takes part in airway inflammation (31). Th17 cells induce asthma by secreting inflammatory factors, including IL-17A, IL-17F, IL-6, and tumor necrosis factor- $\alpha(\mathrm{TNF}-\alpha)$ (21). Tregs are a subgroup of $\mathrm{CD}^{+}$Th cells that secrete IL-10 and support the immune balance. When external allergens stimulate the body, the Th17/Treg balance is broken, and the IL-17A secretion increases, which inhibits the production of IL-10. Meanwhile, the massive secretion of IL-8 induced neutrophils to migrate to the airway and release inflammatory mediators, which aggravate airway inflammation (29). Lathrop et al. found that BMSCs injected via the tail vein could inhibit AHE-induced AHR and neutrophil infiltration, possibly by reducing Th17mediated airway inflammation (21). In mouse models of steroid-resistant asthma, iPSC-MSCs exerted a therapeutic effect on neutrophilic asthma by reducing the Th17 cell count and Th17 cytokines (31).

According to Kang et al., compared with the asthma group, numbers of CD $4+\mathrm{CD} 25+\mathrm{Foxp} 3+$ Tregs was significantly increased in the lung lymph nodes after huMSCs treatment, suggesting that huMSCs reduces OVA-induced allergic airway inflammation, which may be mediated by Treg cells (27). Also, after hPMSCs treatment, the levels of IL-10 and forkhead box P3 (Foxp3) in lymph, peripheral blood, and lung tissue samples were markedly increased, whereas the level of RAR-related orphan receptor gamma (RORgammat) was decreased. These results suggest that hPMSCs may improve AHR and inflammation by regulating the Th17/Treg balance in rats with asthma (29). Dai et al. found ADMSCs could inhibit the increase of IL17 and RORgamma mRNA levels in asthmatic lungs and increase the proportion of CD4+CD25+Foxp3+ Tregs in the spleen, thus attenuating OVA-induced AHR and airway inflammation (24).

\section{Regulatory effect of MSCs on DCs}

DC plays a vital role in maintaining the body's immune response and tolerance and is involved early in pathogenesis of asthma (32). DCs are significantly increased in asthmatic patients, and they are often in an over-mature state. BMSCs inhibit the migration of DCs from lungs to mediastinal lymph nodes and suppress their maturation, reducing the release of Th2 cytokines in the nodules. Also, DCs treated with BMSCs have a lower ability to activate naïve and effector Th2 cells, along with decreased production of chemokine ligand 17 (CCL17). Thus, BMSCs play a therapeutic effect on asthma by inhibiting the excessive maturation and increase of DCs (47).

\section{Regulatory effect of MSCs on macrophages}

Macrophage polarization plays a vital role in the pathogenesis of asthma. In mouse models of CRE-induced acute asthma, BMSCs promoted the switch of macrophage phenotypes from proinflammatory M1 to anti-inflammatory M2. The M1 markers, including IL-6, IL-1 $\beta$, and nitric oxide synthase (NOS2) mRNA levels, were remarkably decreased, while the mRNA levels of M2 markers, including arginase 1 (Arg-1), FIZZ1, and YM-1 were significantly increased. In an in vitro experiment, BMSCs inhibited the expressions of M1 inflammatory factors, increased the expressions of M2 anti-inflammatory factors, and promoted the switch of macrophages to M2; thus, it exerted a therapeutic effect on asthma (49). Song et al. also found that human BMSCs mediated the polarization of alveolar macrophages through TGF- $\beta$ signaling, thus alleviating experimental asthma (46). In an acute mouse model of HDM (Derf)induced asthma, MSCs were labeled with PKH26 fluorescent marker before intravenous injection, and then macrophages phagocyted in vivo the injected MSCs. Only PKH26+ macrophages expressed M2 phenotype, while the innate PKH26- macrophages expressed the M1 phenotype. It was found MSCs attenuated asthma by being phagocyted by lung macrophages, which acquire an M2 suppressive phenotype (18).

\section{Regulatory effect of MSCs on epithelial cells}

Airway epithelial cell injury plays a vital role in activating airway inflammation in allergic asthma. MSCs can differentiate into multiple cellular lineages. In particular, it can promote the repair of airway epithelium and restore its structure and integrity (50). In an in vitro experiment, porcine BMSCs cultured in BEpiCM containing fibroblast growth factor and prostaglandin E2 for 5 or 10 days 
showed the highest expressions of epithelial markers including cytokeratin 7 (CK7), CK8, and epithelial cell adhesion molecules (EpCAM) and functional marker mucin 1 (MUC1). Thus, porcine BMSCs demonstrate a commitment to the epithelial lineage and may facilitate the repair of denuded airway epithelium (50). In another study, the human bronchial epithelial cell line BEAS-2B was induced to undergo apoptosis with a hypoxia mimic of $\mathrm{CoCl}_{2}$ damage. Treatment of iPSC-MSCs significantly decreased apoptosis of BEAS-2B cells, and there was a high miR-21 expression in injured BEAS-2B cells after MSC treatment. Moreover, the protective effect of MSCs on the injured BEAS-2B cells was reversed after the transfection of the miR-21 inhibitor. Thus, iPSC-MSCs protect bronchial epithelial cells from hypoxia-induced injury via miR-21 (51). The increased transfer of mitochondria from BMSCs to epithelial cells can promote the repair of epithelial injury and reduce AHR and remodeling. Overexpression of Miro1 in BSMCs can further enhance epithelial repair to improve asthma via promoting mitochondria transfer, and this therapeutical effect is not associated with their nonspecific paracrine effects (34). Yao et al. found that iPSCMSC transplantation significantly reduced $\mathrm{T}$ helper 2 cytokines, attenuated the mitochondrial dysfunction of epithelial cells, and alleviated asthma inflammation in mice. The primary mechanisms included the tunneling nanotubes (TNTs) formed between iPSC-MSCs and epithelial cells, which enhanced mitochondrial transfer from iPSC-MSCs to epithelial cells via TNTs and promoted the repair of epithelial injury. In particular, connexin 43 (CX43) plays a critical role in regulating TNT formation by mediating mitochondrial transfer between iPSC-MSCs and epithelial cells (52).

\section{Challenges and controversies in MSC-based treatment}

Although the therapeutic effect of MSCs on asthma has been verified in animal models, challenges and controversies in MSC-based clinical treatment persist. First, MSCs need to be cultured in vitro for clinical studies and basic research; however, in vitro culture will affect the regeneration, proliferation, and differentiation of MSCs. Second, MSCs from various sources have different therapeutic effects on asthma, and different routes of administration will also affect the efficacy. Therefore, it is urgent to set up the standard operating procedures for the harvest, culture, and delivery of MSCs. The storage and management of MSCs are also challenging. MSC-CM and MSC-EV on asthma have been proved to have the same therapeutic effects as MSCs in the treatment of asthma. MSCs have an excellent inhibitory effect on airway inflammation in asthma, but its inhibitory effect on airway remodeling is not obvious in chronic asthma. In recent years, pretreatment, or genetic modifications of MSCs and combinations of MSCs with other drugs have become new research directions. At present, the primary mechanism via which MSCs exert its therapeutic effects is by regulating the immune cells, especially $\mathrm{T}$ cells, and its repairing effects on epithelial cells have also been revealed. The specific molecular mechanism of MSCs for asthma therapy is needed to further study.

\section{Conclusions}

Currently, corticosteroids and long-acting $\beta$-agonists are main drugs for asthma therapy. But for refractory asthma and steroid-resistant neutrophilic asthma, drugs can't control the asthma symptom well. Several studies indicated that MSCs suppressed neutrophil-dominant airway inflammation in a murine model of asthma. MSCs might fill the gap in this aspect. With a deeper understanding of the mechanism of MSCs in the next few years, it will help enhance the therapeutic effect of MSCs on asthma in clinical trials and reduce the incidence and mortality of severe refractory asthma. The MSC extracellular vesicles and conditioned medium are easily maintained for quality control and storage; as potentially suitable substitutes for MSCs in future cell-free-based therapeutic approaches, they may help accelerate the use of MSCs in the clinical treatment of asthma.

\section{Acknowledgments}

Funding: This work was supported by the National Natural Science Foundation of China (NSFC:81770028) and the Natural Science Foundation of Guangdong (2018A030310011).

\section{Footnote}

Reporting Checklist: The authors have completed the Narrative Review reporting checklist. Available at http:// dx.doi.org/10.21037/atm-20-6389

Conflicts of Interest: All authors have completed the ICMJE uniform disclosure form (available at http://dx.doi. org/10.21037/atm-20-6389). CQ serves as an unpaid 
editorial board member of Annals of Translational Medicine from Feb 2019 to Jan 2021. The authors have no other conflicts of interest to declare.

Ethical Statement: The authors are accountable for all aspects of the work in ensuring that questions related to the accuracy or integrity of any part of the work are appropriately investigated and resolved.

Open Access Statement: This is an Open Access article distributed in accordance with the Creative Commons Attribution-NonCommercial-NoDerivs 4.0 International License (CC BY-NC-ND 4.0), which permits the noncommercial replication and distribution of the article with the strict proviso that no changes or edits are made and the original work is properly cited (including links to both the formal publication through the relevant DOI and the license). See: https://creativecommons.org/licenses/by-nc-nd/4.0/.

\section{References}

1. Papi A, Brightling C, Pedersen SE, et al. Asthma. Lancet 2018;391:783-800.

2. Wang L, Feng M, Li Q, et al. Advances in nanotechnology and asthma. Ann Transl Med 2019;7:180.

3. Loftus PA, Wise SK. Epidemiology of asthma. Curr Opin Otolaryngol Head Neck Surg 2016;24:245-9.

4. Becker AB, Abrams EM. Asthma guidelines: the Global Initiative for Asthma in relation to national guidelines. Curr Opin Allergy Clin Immunol 2017;17:99-103.

5. Kepil Ozdemir S, Bavbek S. Prospects for new and emerging therapeutics in severe asthma: the role of biologics. Expert Rev Respir Med 2017;11:505-12.

6. Lin J, Xing B, Chen P, et al. Chinese expert consensusbased guideline on assessment and management of asthma exacerbation. J Thorac Dis 2019;11:4918-35.

7. Menzella F, Bertolini F, Biava M, et al. Severe refractory asthma: current treatment options and ongoing research. Drugs Context 2018;7:212561.

8. Golpanian S, Wolf A, Hatzistergos KE, et al. Rebuilding the Damaged Heart: Mesenchymal Stem Cells, CellBased Therapy, and Engineered Heart Tissue. Physiol Rev 2016;96:1127-68.

9. Wang Y, Chen X, Cao W, et al. Plasticity of mesenchymal stem cells in immunomodulation: pathological and therapeutic implications. Nat Immunol 2014;15:1009-16.

10. Gao F, Chiu SM, Motan DA, et al. Mesenchymal stem cells and immunomodulation: current status and future prospects. Cell Death Dis 2016;7:e2062.

11. Padem N, Saltoun C. Classification of asthma. Allergy Asthma Proc 2019;40:385-8.

12. Hancox RJ, Cowan DC, Aldridge RE, et al. Asthma phenotypes: consistency of classification using induced sputum. Respirology 2012;17:461-6.

13. Kianmeher M, Ghorani V, Boskabady MH. Animal Model of Asthma, Various Methods and Measured Parameters: A Methodological Review. Iran J Allergy Asthma Immunol 2016;15:445-65.

14. Firinci F, Karaman M, Baran Y, et al. Mesenchymal stem cells ameliorate the histopathological changes in a murine model of chronic asthma. Int Immunopharmacol 2011;11:1120-6.

15. Ge X, Bai C, Yang J, et al. Effect of mesenchymal stem cells on inhibiting airway remodeling and airway inflammation in chronic asthma. J Cell Biochem 2013;114:1595-605.

16. Ou-Yang HF, Huang Y, Hu XB, et al. Suppression of allergic airway inflammation in a mouse model of asthma by exogenous mesenchymal stem cells. Exp Biol Med (Maywood) 2011;236:1461-7.

17. Goodwin M, Sueblinvong V, Eisenhauer P, et al. Bone marrow-derived mesenchymal stromal cells inhibit Th2mediated allergic airways inflammation in mice. Stem Cells 2011;29:1137-48.

18. Braza F, Dirou S, Forest V, et al. Mesenchymal Stem Cells Induce Suppressive Macrophages Through Phagocytosis in a Mouse Model of Asthma. Stem Cells 2016;34:1836-45.

19. Lee SH, Jang AS, Kwon JH, et al. Mesenchymal stem cell transfer suppresses airway remodeling in a toluene diisocyanate-induced murine asthma model. Allergy Asthma Immunol Res 2011;3:205-11.

20. Nemeth K, Keane-Myers A, Brown JM, et al. Bone marrow stromal cells use TGF-beta to suppress allergic responses in a mouse model of ragweed-induced asthma. Proc Natl Acad Sci U S A 2010;107:5652-7.

21. Lathrop MJ, Brooks EM, Bonenfant NR, et al. Mesenchymal stromal cells mediate Aspergillus hyphal extract-induced allergic airway inflammation by inhibition of the Th17 signaling pathway. Stem Cells Transl Med 2014;3:194-205.

22. Ogulur I, Gurhan G, Aksoy A, et al. Suppressive effect of compact bone-derived mesenchymal stem cells on chronic airway remodeling in murine model of asthma. Int Immunopharmacol 2014;20:101-9.

23. Marinas-Pardo L, Mirones I, Amor-Carro O, et al. 
Mesenchymal stem cells regulate airway contractile tissue remodeling in murine experimental asthma. Allergy 2014;69:730-40.

24. Dai R, Liu J, Cai S, et al. Delivery of adipose-derived mesenchymal stem cells attenuates airway responsiveness and inflammation in a mouse model of ovalbumin-induced asthma. Am J Transl Res 2017;9:2421-8.

25. Martinez-Gonzalez I, Cruz MJ, Moreno R, et al. Human mesenchymal stem cells resolve airway inflammation, hyperreactivity, and histopathology in a mouse model of occupational asthma. Stem Cells Dev 2014;23:2352-63.

26. Chan CK, Lin TC, Huang YA, et al. The modulation of Th2 immune pathway in the immunosuppressive effect of human umbilical cord mesenchymal stem cells in a murine asthmatic model. Inflamm Res 2016;65:795-801.

27. Kang SY, Park DE, Song WJ, et al. Immunologic regulatory effects of human umbilical cord blood-derived mesenchymal stem cells in a murine ovalbumin asthma model. Clin Exp Allergy 2017;47:937-45.

28. Hong GH, Kwon HS, Lee KY, et al. hMSCs suppress neutrophil-dominant airway inflammation in a murine model of asthma. Exp Mol Med 2017;49:e288.

29. Li Y, Li H, Cao Y, et al. Placentaderived mesenchymal stem cells improve airway hyperresponsiveness and inflammation in asthmatic rats by modulating the Th17/ Treg balance. Mol Med Rep 2017;16:8137-45.

30. Royce SG, Rele S, Broughton BRS, et al. Intranasal administration of mesenchymoangioblast-derived mesenchymal stem cells abrogates airway fibrosis and airway hyperresponsiveness associated with chronic allergic airways disease. FASEB J 2017;31:4168-78.

31. Fang SB, Zhang HY, Jiang AY, et al. Human iPSC-MSCs prevent steroid-resistant neutrophilic airway inflammation via modulating Th17 phenotypes. Stem Cell Res Ther 2018;9:147.

32. Barrios RJ, Kheradmand F, Batts L, et al. Asthma: pathology and pathophysiology. Arch Pathol Lab Med 2006;130:447-51.

33. Abreu SC, Antunes MA, Xisto DG, et al. Bone Marrow, Adipose, and Lung Tissue-Derived Murine Mesenchymal Stromal Cells Release Different Mediators and Differentially Affect Airway and Lung Parenchyma in Experimental Asthma. Stem Cells Transl Med 2017;6:1557-67.

34. Ahmad T, Mukherjee S, Pattnaik B, et al. Miro1 regulates intercellular mitochondrial transport \& enhances mesenchymal stem cell rescue efficacy. EMBO J 2014;33:994-1010.
35. Han XP, Zhang FQ, Tan XS, et al. EPO modified MSCs can inhibit asthmatic airway remodeling in an animal model. J Cell Biochem 2018;119:1008-16.

36. Abreu SC, Lopes-Pacheco M, da Silva AL, et al. Eicosapentaenoic Acid Enhances the Effects of Mesenchymal Stromal Cell Therapy in Experimental Allergic Asthma. Front Immunol 2018;9:1147.

37. Kwak J, Choi SJ, Oh W, et al. Cobalt Chloride Enhances the Anti-Inflammatory Potency of Human Umbilical Cord Blood-Derived Mesenchymal Stem Cells through the ERK-HIF-1alpha-MicroRNA-146a-Mediated Signaling Pathway. Stem Cells Int 2018;2018:4978763.

38. Abreu SC, Xisto DG, de Oliveira TB, et al. Serum from Asthmatic Mice Potentiates the Therapeutic Effects of Mesenchymal Stromal Cells in Experimental Allergic Asthma. Stem Cells Transl Med 2019;8:301-12.

39. Cruz FF, Borg ZD, Goodwin M, et al. Systemic Administration of Human Bone Marrow-Derived Mesenchymal Stromal Cell Extracellular Vesicles Ameliorates Aspergillus Hyphal Extract-Induced Allergic Airway Inflammation in Immunocompetent Mice. Stem Cells Transl Med 2015;4:1302-16.

40. Keyhanmanesh R, Rahbarghazi R, Aslani MR, et al. Systemic delivery of mesenchymal stem cells condition media in repeated doses acts as magic bullets in restoring IFN-gamma/IL-4 balance in asthmatic rats. Life Sci 2018;212:30-6.

41. Royce SG, Shen M, Patel KP, et al. Mesenchymal stem cells and serelaxin synergistically abrogate established airway fibrosis in an experimental model of chronic allergic airways disease. Stem Cell Res 2015;15:495-505.

42. Inamdar AC, Inamdar AA. Mesenchymal stem cell therapy in lung disorders: pathogenesis of lung diseases and mechanism of action of mesenchymal stem cell. Exp Lung Res 2013;39:315-27.

43. Gao P, Zhou Y, Xian L, et al. Functional effects of TGFbeta 1 on mesenchymal stem cell mobilization in cockroach allergen-induced asthma. J Immunol 2014;192:4560-70.

44. Xu T, Zhou Y, Qiu L, et al. Aryl Hydrocarbon Receptor Protects Lungs from Cockroach Allergen-Induced Inflammation by Modulating Mesenchymal Stem Cells. J Immunol 2015;195:5539-50.

45. Cruz FF, Borg ZD, Goodwin M, et al. CD11b+ and Sca1+ Cells Exert the Main Beneficial Effects of Systemically Administered Bone Marrow-Derived Mononuclear Cells in a Murine Model of Mixed Th2/Th17 Allergic Airway Inflammation. Stem Cells Transl Med 2016;5:488-99.

46. Song X, Xie S, Lu K, et al. Mesenchymal stem cells 
alleviate experimental asthma by inducing polarization of alveolar macrophages. Inflammation 2015;38:485-92.

47. Zeng SL, Wang LH, Li P, et al. Mesenchymal stem cells abrogate experimental asthma by altering dendritic cell function. Mol Med Rep 2015;12:2511-20.

48. Ke X, Do DC, Li C, et al. Ras homolog family member A/Rho-associated protein kinase 1 signaling modulates lineage commitment of mesenchymal stem cells in asthmatic patients through lymphoid enhancer-binding factor 1. J Allergy Clin Immunol 2019;143:1560-74.e6.

49. Cui Z, Feng Y, Li D, et al. Activation of aryl hydrocarbon receptor (AhR) in mesenchymal stem cells modulates macrophage polarization in asthma. J Immunotoxicol

Cite this article as: Yu X, Yu L, Guo B, Chen R, Qiu C. Research advances in mesenchymal stem cell therapy for asthma. Ann Transl Med 2020;8(21):1461. doi: 10.21037/atm-206389
2020;17:21-30.

50. Kokubun K, Pankajakshan D, Kim MJ, et al. Differentiation of porcine mesenchymal stem cells into epithelial cells as a potential therapeutic application to facilitate epithelial regeneration. J Tissue Eng Regen Med 2016;10:E73-83.

51. Li CL, Xu ZB, Fan XL, et al. MicroRNA-21 Mediates the Protective Effects of Mesenchymal Stem Cells Derived from iPSCs to Human Bronchial Epithelial Cell Injury Under Hypoxia. Cell Transplant 2018;27:571-83.

52. Yao Y, Fan XL, Jiang D, et al. Connexin 43-Mediated Mitochondrial Transfer of iPSC-MSCs Alleviates Asthma Inflammation. Stem Cell Reports 2018;11:1120-35. 